\title{
Die Anwendung von Farbstoffgemischen als Hilfsmittel in der Mikroskopie.
}

\author{
Von \\ P. P, Fechner.
}

Mitteilungen aus dem Forschungs- und Uniersuchungslaboratorium der . Studien-und Treuhand-Gesellschaft für Getreide-Müllerei m. b. H. in $D$ ïs seld orf. (Leiter: E. Meyer, Nahrungsmittelchemiker.)

[Eingegangen am 24. Februar 1921.]

\section{Analysen von Brot-, Mehl- und Futtermittelproben mittels Färbung durch "Violett".}

In neuerer Zeit hat die Untersuchung von Backwaren, Mehlen und Futtermitteln an Bedeutung zugenommen. Die Bäcker werden zur Herstellung von Brot mit Meblen verschiedener Getreidearten beliefert, von denen augenblicklich fast ein Drittel zur Streckung dienen. So werden Brote aus einer Mischung von Roggen, Weizen, Gerste, Kartuffel, Mais usw. in vorgeschriebener Zusammensetzung hergestellt.

Für einen einigermaßen geübten Mikroskopiker ist es nicht schwer festzustellen, aus welchen Mehlen das Brot gebacken ist, dagegen erfordert eine Bestimmung der prozentualen Zusammensetzung längere Übung und größte Sorgfalt.

Als Hilfsmittel zur Auffindung und Auszählung der im Präparat befindlichen Elemente bezw. zur Feststellung der Zugehörigkeit zur betreffenden Art ist schon früher von verschiedenen Seiten die Anwendung von Farbstoffen bezw. Farbstoffgemischen empfohlen worden. So stellte W. Herter Anfang des Jahres 1915 ) ein Farbgemisch Schwarz-Weiß-Rot her, welches für Brotuntersuchungen große Dienste leistete. Scheffer und Posener erzielten 1915 ähnliche Ergebnisse in der Differentialfärbung des K-Brotes. Bei meinen Versuchen ist es mir gelungen, ein gleiches Farbgemisch wie W. Herter herzustellen, worüber ich nachfolgend einen kurzen Bericht gebe. Letztgenanntes Farbgemisch verwendet man hauptsächlich für die stärkebaltigen Präparate (Gebäck-, Mehl- und Futternittelproben), die man noch nicht einer Anreicherung unterworfen hat.

Ein wesentlich anderes Farbgemisch stellt aber mein Farbenreagens „Violet $t^{4 "}$ ) dar. Es eignet sich in erster Linie für die Untersuchung der Probe selbst.

\section{Qualitative Analyse.}

a) Untersuchung der Probe.

Das Präparat wird wie gewöhnlich hergestellt, indem man von dem zu untersuchenden Gegenstand soviel nimmt, wie die Größe eines Pfefferkornes ausmacht. Hierzu fügt man statt des Wassers oder anderer Mikroskopierflüssigkeiten 2-3 Tropfen Violett hinzu. Man achte hierbei nur darauf, daß das Präparat nicht zu stark ist und die Teilchen auf dem Objektträger gut verteilt und mit der Farbe gleichmäßig ver-

1) W. Herter, Dis quantitative Bestimmung der verkleisterten Kartoffelstärke (der ,Kleisterzellen") im Brot. - Zeitschr. ges. Getreidewesen 1915, 7, Nr. 2.

7) Die Farbemische sind in Flasehen zu N. 20,- durch das hiesige Laboratorium zu beziehen. 
mischt sind. Hierauf überläbt man das Präparat etwa 5 Minuten der Ruhe, um ein möglichst gutes Resultat in der Färbung der verschiedenen Teile zu erzielen.

Die Färbungen der einzelnen Elemente des Untersuchungsobjektes sind folgende:

I. Blaugrün bis blauviolefl: Aus Cellulose bestehende Zellwände, wie Längs-, Quer-, Schlauchzellen, Haare, leere Aleuronzellen und das Gewebe des Keim. lings. Auch die Speicher- oder Kleisterzellen, die je nach den Grade der Verkleistermng der in innen enthaltenen Stäke schwach blau-violett bis blau erscheinen.

II. Violet bis rötlieh: Eiweiß, z. B. der in Mehlkörper des Getreides enthaltene Kleber und auch junges Zellgewebe.

III. Gelb bis helluraun: Fette, Öle; so z, B, die Tröpfchen in den Aleuronzellen.

IV. Weiß oder farblos: Stärkekörner aller Art.

Auch sonst schwach mikroskopisch sichtbare Körper, wie Schimmelmycel, nehmen Farbe auf; wenn auch nicht eine ausgesprochene Färbung eintritt, so fallen sie doch durch einen dunkleren Farbenton stärker auf.

Die Differenz in der Färbung ermöglicht so ein schnelles und leichtes Auffinden von oft in sehr geringer Menge im Präparat vorhandenen Elementen.

b) Untersuchung der Anrejcherung.

Die Anreicherung wird, wie schon in der "Zeitschrift für das gesamte Getreidewesen"1) veröffentlicht ist, durch Aufkochen der zu untersuchenden Probe mit 1\%-iger Schwefelsäure und Übergießen des Rückstandes mit ebenso verdünnter Natronlauge hergestellt. Genaueres daräber siehe: Brotuntersuchungen mit dem Farbgemisch SchwarzWeiß-Rot von Dr. W. Herter.

Bei der Herstellung des Präparates verfährt man wie bei der Untersuchung der ursprünglichen Probe. Im mikroskopischen Bilde treten nunmehr die Farbentöne der einzelnen Elemente deutlicher hervor, da durch das Anreicherungsverfahren das zu untersuchende Objekt von der Stärke befreit ist.

\section{Quantitative Analyse.}

Zur Ermittlung des Mengenverbältnisses der einzelnen Bestaudteile zählt man zunächst die Querzellen der Getreidearten aus und berechnet danach die prozentuale Zusammensetzung, weiterhin schätzt man das Verhältnis der einzelnen Stärkearten unter genauer Berücksichtigung ihres Volumens. Über diese letztere Methode sei auf die Abhandlung von W. Herter in dieser Zeitschrift ${ }^{2}$ ) verwiesen.

Untersuchungen von Mehlen und Futtermitteln sind in gleicher Weise auszuführen.

Empfehlenswert dürtte es sein, neben vorstehender Methode W. Herter's mein Farbengemisch "Schwarz-Weiß-Rot" anzuwenden, um eine Bestätigung bezw. Erweiterung des Ergebnisses zu erhalten.

\section{Farbengemisch „Schwarz-Weik-Rot" zmm Nachweise von Kartoffeln im Brot.}

Bei meinen letzten Versuchen ist es mir gelungen, ein Farbengemisch herzustellen, das sich in gleicher Weize wie das "Schwarz-Weiß-Rot" von W. Herter für Brot-

ग) Zeitschr. f. d. gesamte Getreidewesen 1917, 9, Nr. 2.

2) Diese $/ / /$ eitschrift $1919,: 38,65$. 
unterşuchungen, hauptsächlich für den Nachweis von Kartoffelstärke im Brot, eignet. Es unterscheidet sich von diesem nur durch ein stärkeres Hervortreten der Farbenunterschiede. Die Farbentöne sind die des Herter'schen Schwarz-Weiß-Rot, nämlich:

I. Schwarz bi braun: Stärkekörner der Gramineen (Roggen, Weizen, Gerste, Hafer, Reis, Hirse, Mais) sowie des Buchweizens.

II. Weib und stark lichtbrechend: Stärkeköner der Palmen und anderer Monokotyledonen (Sago, Tacea, Banane usw., der Kartoffel und in geringerem Grade der Edelkastanie, der Roßkastanie, der Eichel, der Leguminosen, der Batate and des Mondioca.

III. Rot: Aus reiner Cellulose bestehende Zellmembranen, vor allem die Speicheroder Kleisterzellen, die je nach dem Grade der Verkleisterung der in ihnen enthaltenen Stärke schwach rosa umrandet bis tief purpurrot erscheinen. Ferner jugendliche Gewebe der Zellen des Keimlings, ebenso Stärke der Aleuron- und der hyalinen Schicht beim Getreide.

Das Anfertigen der Präparate geschieht in der gebräuchlichen Weise:

Ein Krümchen in der Größe einer halben Erbse von dem zu untersuchenden Brot wird auf den Objektträger gebracht und mit Wasser etwas angefeuchtet, sodaß es sich gut zu einer breilgen Flüssigkeit verreiben läbt. Hierauf setzt man von dem Farbengemisch etwas hinzu und vermischt es gut mit der aufgeweichten Brotmasse.

Es ist auch hierbei ratsam ein nicht zu starkes Präparat anzufertigen und auf gute Verteilung der Substanz auf dem Objektträger zu achten.

Ausführlich hat darüber Herter ${ }^{1}$ ) berichtet.

2) Zeitschr. f. d. gesamte Getreidewesen 1917, 9, Nr. 2.

\section{Über Maniokmehl.}

Von

\section{Dr. Olemens Orimme.}

Mitteilung aus dem Institut fär angewandte Botanik in Hamburg.

[Eingcgangen. an 12, März 1921.]

Die Maniokpflanze ist ursprünglich in Brasilien beheimatet, doch findet man sie jetzt in vielen Kulturvarietäten in fast allen tropischen und subtropischen Regionen der ganzen Erde. Für die menschliche und tierische Ernährung und die technische Ausnutzung kommen vor allem zwei Arten in Frage: Manihot utilissima Pohl (= Manihot edulis Plumier, Jatropha Manihot L, Janipha Manihot Kunth), der sog. bittere Maniok, welcher in allen grünen Teilen und in der Wurzel merkliche Mengen Blausäure enthält, und Manihot palmata Mueller (= Manihot Aipi Pohl, Manihot dulcis Baillon, Jatropha dulcis Rottboel), der sog. süße Maniok, welcher praktisch blausäurefrei ist.

Botanisch gehören die Maniokarten zur Familie der Euphorbiaceen. Sie bilden Sträucher oder Bäumchen mit weílichen, leicht zerbrechlichen, mehr oder minder ge- 